\title{
BUILDING MODERN VIBRATION DIAGNOSTICS SYSTEMS BASED ON THE FREQUENCY-TIME TRANSFORMATIONS OF A MEASURED SIGNAL
}

\author{
Vasikh Yasoveev, Marat Akhmetshin ${ }^{\mathrm{a}}$ \\ Ufa State Aviation Technical University (USATU), 450008, Ufa, Russia
}

\begin{abstract}
Basic methods of analysis of vibration transducers signals were reviewed. Continuous wavelet transform, being a time-frequency transform, was found to be an advanced mathematical tool for analysis of vibration signals. Experimental studies revealed obvious changes in the continuous wavelet transform spectrum depending on the existing defects. A method for detection and identification of technological violations based on the analysis of CWT spectrum components and normalized correlation coefficient was suggested. In accordance with the suggested method software for vibration diagnostics was developed.
\end{abstract}

\section{Introduction}

At present industrial testing and technological equipment and systems have reached a high level. It affects not only the cost and complexity of the equipment itself but also the ability to be qualitatively repaired without costly and labor-consuming operations.

Vibration is the most informative indicator in terms of health assessment of virtually any class of equipment. However, vibration testing requires that issues of selection of the necessary technical devices should be seriously addressed, as well as revealing diagnostic indicators for each specific class of equipment.

\section{Problem statement}

Development of effective systems for non-destructive and non-disassembling monitoring of technological violations and item's defects is a task of exceptional importance, and the possibility to quickly and adequately identify incorrect modes and deviations of the item's parameters from normalized ones is a priority trend.

\section{State of the art and relevance}

Systems for monitoring and control of vibration testing [1] used nowadays to process a measured signal generally employ a spectral analysis method involving direct Fourier transform (FT), which has a number of limitations and disadvantages. FT does not provide

\footnotetext{
${ }^{a}$ Corresponding author: ahmaratyan@rambler.ru
} 
localization of the analyzed signal in a time area, since the eigenfunction of the Fourier transform is a sine wave that is determined over the whole time interval and, consequently, FT does not take into account changing of the analyzed signal frequency over time.

Wavelet analysis is a special type of linear transformation of signals and physical data $[2,3]$. The basis of eigenfunctions employed by signals decomposition has many specific properties and possibilities. Wavelet functions of the basis allow focusing attention on local peculiarities of the analyzed processes that cannot be revealed by traditional Fourier and Laplace transforms. For instance, fields of various physical parameters of natural environments belong to such processes in geophysics. This applies especially to fields of temperature, pressure, and seismic path profiles, as well as other physical values.

Wavelet transform of a one-dimensional signal [4] is a representation of this signal as a generalized Fourier series or integral by a combination of the basis functions

$$
\psi_{a b}(t)=\frac{1}{\sqrt{a}} \psi\left(\frac{t-b}{a}\right),
$$

constructed from mother (prototype) wavelet $\psi(t)$ which has certain properties due to time shift operations $(b)$ and time scale change $(a)$. Multiplication factor $1 / \sqrt{a}$ provides independence of these functions norm from scale value $a$.

As parameter $a$ increases, the frequency resolution increases and the time resolution decreases, and vice versa, as parameter $a$ decreases, the frequency resolution decreases and the time resolution increases. Therefore, WT is an adaptive transform. Flexible timefrequency window extracts both low frequency and high frequency signal characteristics equally well. This WT feature is a big advantage for analysis of signal local properties.

\section{Methods}

It was suggested to employ the mathematical apparatus of continuous wavelet transform (CWT) to diagnose vibration parameters. In order to reveal CWT spectrum behavior, an experimental study was performed [5], and models of main intrinsic defects and technological violations were defined and analyzed.

By processing and continuous wavelet transforming of a data array obtained during experimental testing with the help of an application operating under MatLab 7.11.0, signals skeletons and CWT results in the form of 3D surfaces were obtained.

During analysis of the skeletons and CWT results' surfaces it was found that manifestation of each analyzed defect type depends on the vibration frequency.

Frequencies for every defect type at which the defect manifests were identified, as well as the CWT spectrum components that are informative for each defect type. These components generally have areas of local maxima.

Figures 1 and 2 demonstrate the difference between the signal skeleton under normal mode and the one when defect No.1 emerges at the testing frequency of $500 \mathrm{~Hz}$. Thus, when the defect is developed up to $50 \%$ of the maximum value, it manifests at $100 \mathrm{~Hz}$ and $500 \mathrm{~Hz}$; and for testing frequency of $500 \mathrm{~Hz}$ the informative spectrum components are $\mathrm{a}=8 . .25$ wherein the emergence of local maxima is observed. 


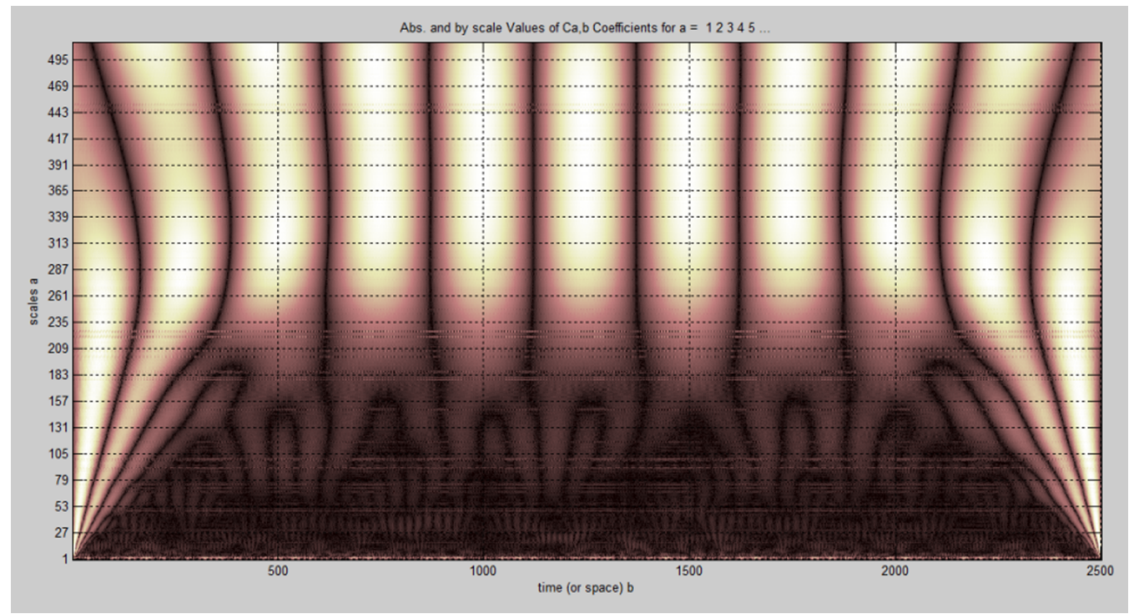

Figure 1. $500 \mathrm{~Hz}$ signal skeleton under normal model.

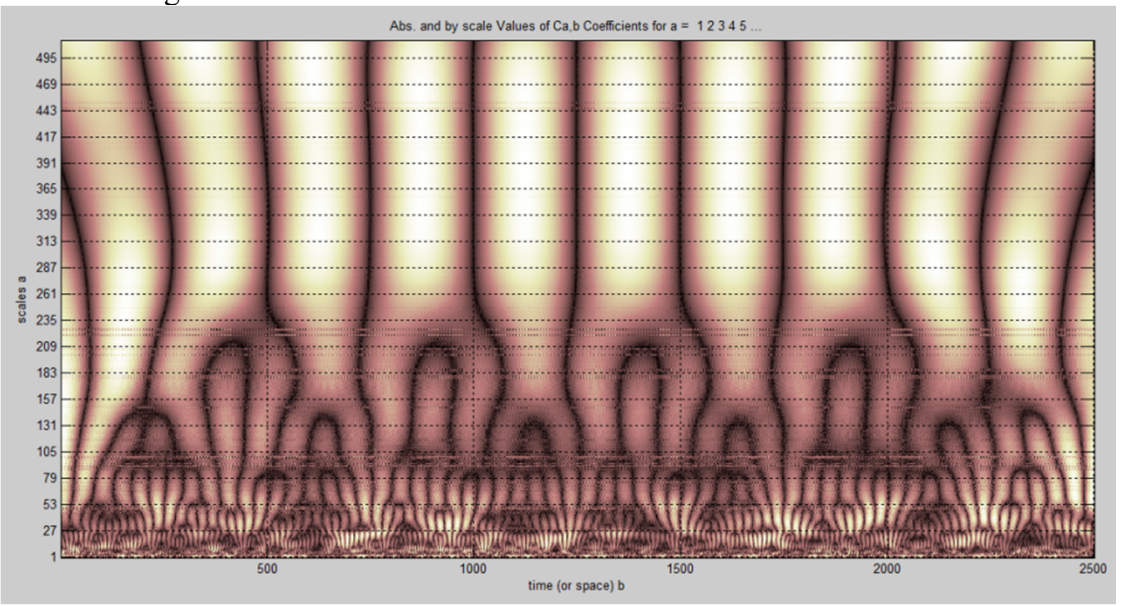

Figure 2. $500 \mathrm{~Hz}$ signal skeleton for defect No.1.

Figure 3 shows the 3D surface model of CWT results for the signal frequency of $1 \mathrm{kHz}$. It is clearly seen that $\mathrm{a}=150 . .200$ for the carrier frequency. Emergence of defect No. 2 is characterized by emergence of high frequency components of the wavelet spectrum when $\mathrm{a}<80$.

For each defect type the autocorrelation function (AF) and intercorrelation function (IF) were defined. During evaluation of the autocorrelation function of the analyzed signals it was determined that the maximal normalized correlation coefficient is approximately equal to 1 when the relative shift equals zero. Therefore, we can claim that the analyzed signals have such property as ergodicity, and it is sufficient to perform analysis using values obtained from one period of testing. The minimum signal sampling duration for analysis is $0.02 \mathrm{~s}$. 


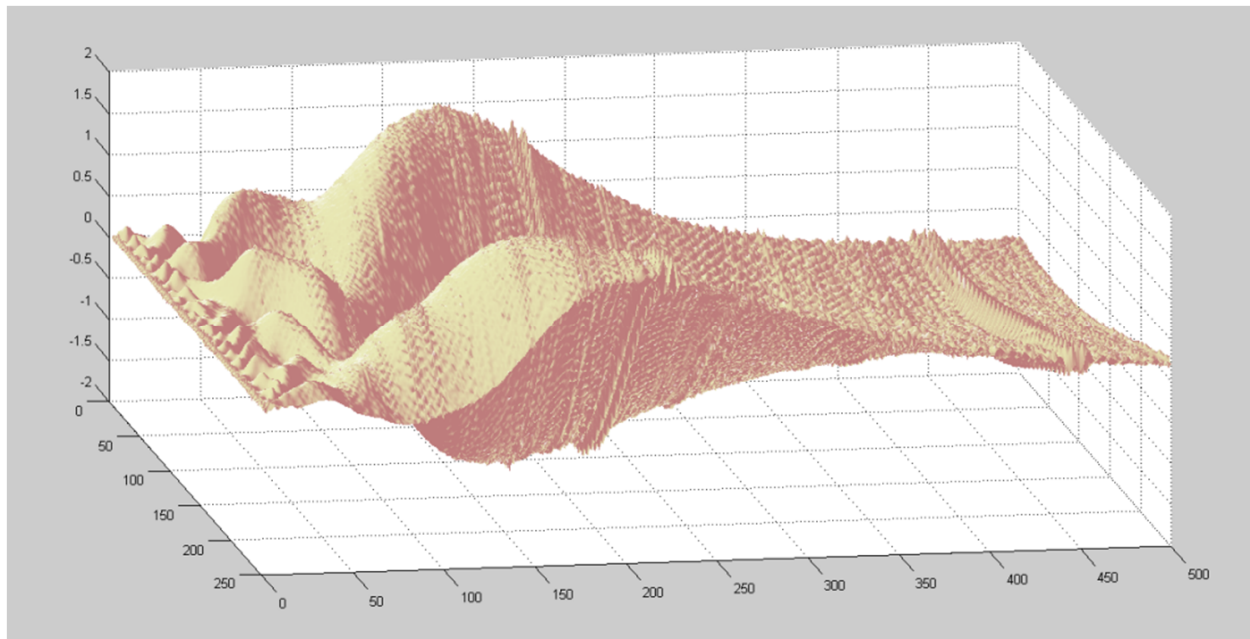

Figure 3. 3D surface model of CWT results for the signal frequency of $1 \mathrm{kHz}$.

During correlation analysis of informative spectrum components for each defect type it was determined that maximal normalized correlation coefficient equals 0.75 . It means that defect modes (modes in which defects were detected) can always be identified correctly. There is no correlation between the defects as they manifest on different CWT spectrum components. Figure 5 shows graph of intercorrelation function of informative CWT spectrum component for defect No. 1.

\section{Discussion and application of results}

During analysis of the informative CWT spectrum components, rules for diagnostics and criteria for determination and identification of the defects based on evaluation of local maxima values and maximal normalized correlation coefficient were defined.

An algorithm for identification and determination of the existing defects and technological violations was developed in accordance with the rules for diagnostics. Figure 4 shows a typical cycle of the algorithm. 


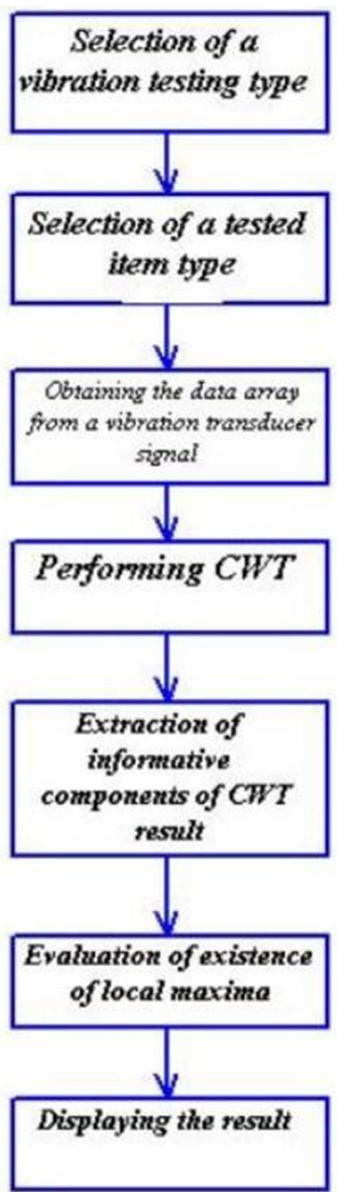

Figure 4. Cycle of the algorithm for identification and determination of the existing defects and technological violations.

This algorithm is the basis of the developed application software whose interface is illustrated in Figure 5. 


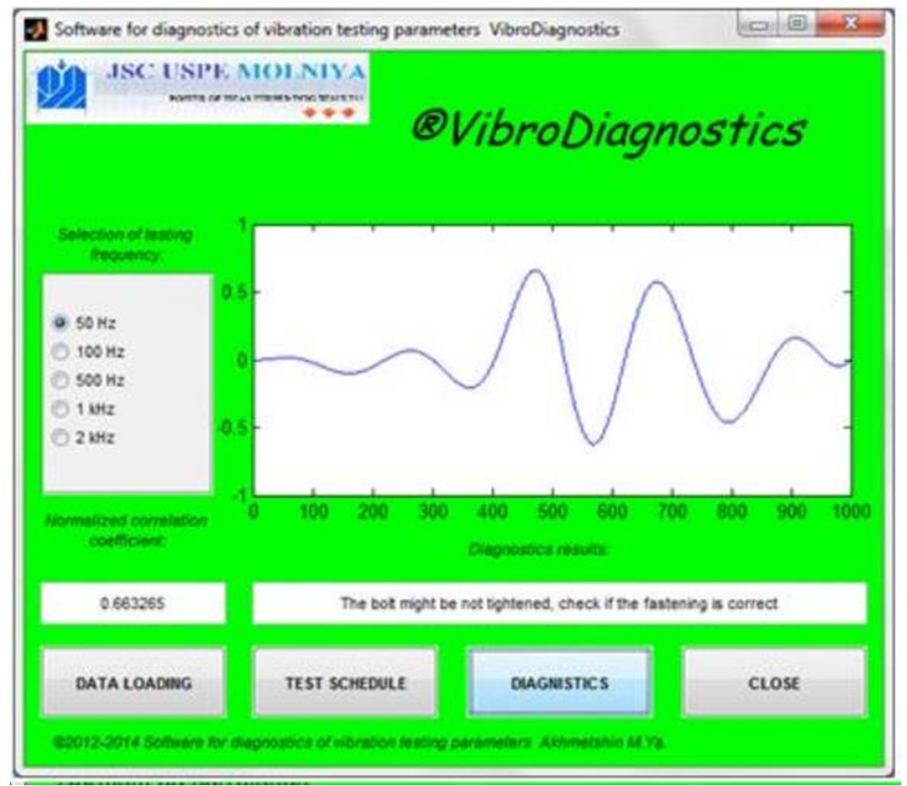

Figure 5. Interface of the developed software for vibration diagnostics.

The software makes it possible to load the mat or .xls files of the analyzed signals from a computer hard disc, look through the loaded signals graphs, as well as the interconnection function graph. The software interface has round buttons to select testing frequency. Clicking DIAGNOSTICS button starts the main program the operation of which is followed by displaying the context message on diagnostics results and correlation coefficient value, as well as the intercorrelation function graph.

\section{Conclusions}

Following the study of the existing methods to analyze measured signals in modern vibration diagnostics systems, methods have been developed enabling to analyze signals and their changes not only in terms of their frequency, but also in a time scale. An opportunity has emerged to assess the effects of ambient conditions on the structure of the analyzed signals, in particular, vibration signals.

In the course of the experimental study, CWT spectrum changes have been revealed and information components of CWT spectrum corresponding to each defect type have been defined. The correlation analysis of the studied signals has shown that each defect type can be correctly identified.

Rules for diagnostics and criteria for determination and identification of the defects based on evaluation of local maxima values and maximal normalized correlation coefficient, which became the basis of the vibration diagnostics application algorithm, have been defined.

Analysis of the signals of a time area makes it possible to predict and to early detect defects still barely manifested, which has a large practical significance when developing modern vibration diagnostics systems. The developed method will also be useful for the development of highly effective neural network algorithms for processing not only vibration signals but also any other complex measured signals requiring consequent frequency-time analysis. 


\section{References}

[1] L. Debnath, International Journal of Mathematical Education in Science and Technology 29, 677 (1998) doi: 10.1080/0020739980290504

[2] C. Chiann, P. A. Morettin, Journal of Nonparametric Statistics 10 , 1 (1999)

[3] M. C. Hu, I. S. Tsai, The Journal of The Textile Institute 91, 420 (2000)

[4] L. Shao, X. Lin, X. Shao, Applied Spectroscopy Reviews 37, 429 (2002) doi: 10.1081/ASR-120016391

[5] M. Ya. Akhmetshin, V. Kh. Yasoveev, Pribory (8), 33 (2014) 